\author{
K. D. Cole ${ }^{1}$ \\ Mechanical and Materials Engineering, \\ University of Nebraska-Lincoln, \\ W342 Nebraska Hall, \\ Lincoln, NE 65588-0656 \\ e-mail: kcole1@unl.edu \\ B. Cetin \\ Mechanical Engineering, \\ Bilkent University, \\ Bilkent, Ankara 06800, Turkey \\ Y. Demirel \\ Chemical and Biomolecular Engineering, \\ University of Nebraska-Lincoln, \\ Lincoln, NE 68588-0643
}

\section{Semi-Analytical Source Method for Reaction-Diffusion Problems}

Estimation of thermal properties, diffusion properties, or chemical-reaction rates from transient data requires that a model is available that is physically meaningful and suitably precise. The model must also produce numerical values rapidly enough to accommodate iterative regression, inverse methods, or other estimation procedures during which the model is evaluated again and again. Applications that motivate the present work include process control of microreactors, measurement of diffusion properties in microfuel cells, and measurement of reaction kinetics in biological systems. This study introduces a solution method for nonisothermal reaction-diffusion $(R D)$ problems that provides numerical results at high precision and low computation time, especially for calculations of a repetitive nature. Here, the coupled heat and mass balance equations are solved by treating the coupling terms as source terms, so that the solution for concentration and temperature may be cast as integral equations using Green's functions (GF). This new method requires far fewer discretization elements in space and time than fully numeric methods at comparable accuracy. The method is validated by comparison with a benchmark heat transfer solution and a commercial code. Results are presented for a first-order chemical reaction that represents synthesis of vinyl chloride.

[DOI: 10.1115/1.4038987]

Keywords: heat transfer, mass transfer, nonlinear partial differential equation, crossdependence, exact Green's function, piecewise-constant source

\section{Introduction}

Nonisothermal reaction-diffusion (RD) systems describe many transport and rate processes in physical, chemical, and biological systems [1]. Although the motivation is the RD problem, the primary thrust of this paper is to introduce the semi-analytical source (SAS) method for such problems. In this approach, the crossdependence and nonlinear terms in the differential equations describing the RD problem are treated as source terms, and the boundary value problem is recast into an integral equation using Green's functions (GF) for diffusion. There are several distinct advantages to this approach. GF are analytical expressions that exactly satisfy the boundary conditions for concentration and temperature. These GF then serve as physics-based spline functions that exactly fit the problem of interest. In this way, results of comparable accuracy may be attained with far fewer discretization elements in time and space than with a fully numeric solution (such as finite difference (FD) and finite element). As the computation cost for fully numeric solutions scales as the cube of the spatial mesh (order $\mathrm{M}^{3}$ ), the potential for computational savings is substantial. The GF may be computed ahead of time and stored for rapid retrieval, a particular advantage for computations of a repetitive nature, for example in control of industrial processes and for inverse problems associated with indirect measurements.

Next, the pertinent literature will be discussed in two partsfirst that of reaction-diffusion problems in general and then that of semi-analytical methods for solving such problems. Much has been published on mathematically coupled nonlinear differential equations of chemical reaction and diffusion systems by neglecting the possible thermodynamic couplings among heat and mass fluxes, and reaction velocities. Here, thermodynamic coupling refers to induced effects of Soret and Dufour [1-5] that may be considerable in small scale systems due to the presence of large gradients of temperature and concentration. A coupled RD system

\footnotetext{
${ }^{1}$ Corresponding author

Contributed by the Heat Transfer Division of ASME for publication in the Journal of Heat Transfer. Manuscript received August 25, 2017; final manuscript received December 15, 2017; published online April 11, 2018. Assoc. Editor: Alan McGaughey.
}

may require a thorough analysis that takes into account the induced cross effects especially in small-scale structures [4-6]. One of the approaches to describe such a thermodynamically coupled RD system is the nonequilibrium thermodynamic model, which does not require the detailed mechanism of coupling [7].

Some of the well-known RD systems include spread of an epidemic, Lotka-Volterra type of competition-diffusion, BelousovZhabotinskii reaction, and three-component models of quadratic solutions [8]. As Turing [9] showed, a RD system with appropriate nonlinear kinetics can generate stable concentration patterns. Serna et al. [10] studied Turing patterns under nonisothermal RD conditions using the Gray-Scott model. Such patterns may be stationary or oscillatory and may have potential applications in biological morphogenesis; for example, blood clotting can be considered as the formation of localized patterns [11,12]. The respiratory electron transport chain in the inner membrane of mitochondria creates a proton motive force across the membrane, which is used in the endothermic reaction synthesis of adenosine triphosphate. Consequently, the hydrolysis of adenosine triphosphate releases energy used in osmotic work of primary active transport and other mechanical work [13-15].

Modeling the evolution dynamics of infectious diseases requires the mechanism of transmission of the contagion. Mathematical modeling can describe a finite number of subpopulations with spatial densities, whose evolution in time requires nonlinear partial differential equations of RD systems [16]. Elias and Clairambault [17] solved the nonlinear partial differential equations of RD systems for spatio-temporal intracellular protein networks by using semi-implicit Rothe method with the Kedem-Katchalsky boundary conditions. Some exact solution for RD systems also exist $[8,18]$. Tuncer et al. [19] used finite element method to solve RD systems on stationary spheroidal surfaces with possible applications such as in wound healing, tissue regeneration, and cell mobility.

The literature of semi-analytical solutions applied to heat transfer is discussed next. The concept of treating nonlinear terms in the differential equation as source terms is not new, as it was mentioned in 1979 by Stakgold [20]. Taigbenu [21] used the steadyspatial GF as the spatial shape function for a finite element 
solution to a transient heat transfer problem, and the nonlinear portion of the equation was treated as a source term. Jones and Solovjov [22] studied the transient response of a radiation thermometer in which the radiation boundary was modeled as a nonlinear source in their Green's function formulation of the problem. Flint et al. [23] used a GF formulation for a moving volumetric heat source in a parallelepiped to simulate electron-beam welding.

Johansson and Lesnic [24] applied the method of fundamental solutions to the one-dimensional heat equation. In this method, infinite-space GF are placed outside the physical domain with source strengths chosen by collocation to satisfy the boundary conditions. Regularization is needed to compute the source strengths as the matrix solution for source strengths is ill-posed, and the level of ill-posedness depends on the distance between the source points and the boundary. Dong [25] extended the method of fundamental solutions to the heat equation to irregularly shaped two-dimensional domains with internal sources by placing additional source points inside the domain. A weakness of the method is that the results are sensitive to the source-point locations, and the source strength calculation is ill-posed, requiring regularization for numerical stability. Yan et al. [26] extended this method to the three dimensional parallelepiped. Tikhonov regularization was used in the inverse problem for finding the source strengths.

There has been some work involving finite-body diffusion GF that satisfy boundary conditions. Axelsson et al. [27] solved a steady convective-diffusion problem in a rectangular domain using GF which satisfy Dirichlet boundary conditions and have the form of a series. The series coefficients for the GF are found using a Galerkin scheme with matching carried out at grid locations in the domain. Mandaliya et al. [28] treated rectangular and cylindrical geometries for a steady reaction-diffusion problem using GF that satisfy type 3 boundary conditions. In contrast, the present work addresses transient problems.

Lugo-Mendez et al. [29] used effective properties to describe the microscale contribution of pores to mesoscale nonlinear diffusion. The problem formulation involves nonlinear terms in the differential equation which are simulated by treating them as source terms. Using commercial software, computational results are given for the method applied to unit-cell geometries in two and three dimensions. The authors also formulate the problem with GF that satisfy Dirichlet boundary conditions; however, no computations are carried out with this. The authors anticipate that this approach carries a computational burden if the GF is computed fully numerically.

In contrast to Lugo-Mendez et al. [29], in the present work the GF are computed analytically from algorithms that have been verified for high accuracy and optimized for computational efficiency [30]. Nonlinear terms in the differential equation are treated as source terms distributed throughout the domain. Because the boundary conditions are exactly satisfied, no matrix solution is needed for determining source strengths.

A brief outline of the paper is given next. Section 2 describes the mass and energy balances for the reaction-diffusion problem with thermodynamic coupling. Section 3 introduces the semianalytical source method. Section 4 describes a comparison with an available analytic solution to validate the new method. Section 5 gives numerical results for a specific reaction-diffusion problem. Section 6 discusses the scope of additional problems to which the semi-analytical source method may apply, and Sec. 7 contains the summary and conclusions.

\section{Problem Statement}

Assuming that the RD problem is not far from global equilibrium, the following relations for mass flux $J$ and heat flux $J_{q}$ include the effect of thermodynamic coupling:

$$
\begin{aligned}
-J & =D_{e} \nabla C+D_{S} \nabla T \\
-J_{q} & =D_{D} \nabla C+\lambda \nabla T
\end{aligned}
$$

Here, $C$ is concentration and $T$ is temperature. Quantities $D_{e}$ and $\lambda$ are the diffusivity and thermal conductivity, respectively. Thermodynamic coupling is represented by quantities $D_{D}$ (Dufour effect) and $D_{S}$ (Soret effect), which allow for additional mass flux associated with temperature gradient and additional heat flux associated with concentration gradient. The reaction-diffusion problem under discussion is found by combining the above flux expressions with mass and energy balances [31]

$$
\begin{gathered}
\frac{\partial C}{\partial \tau}=D_{e} \frac{\partial^{2} C}{\partial \xi^{2}}+D_{S} \frac{\partial^{2} T}{\partial \xi^{2}}-A_{0} C e^{-E /(R T)} \\
\rho c_{p} \frac{\partial T}{\partial \tau}=\lambda \frac{\partial^{2} T}{\partial \xi^{2}}+D_{D} \frac{\partial^{2} C}{\partial \xi^{2}}+(-\Delta H) A_{0} C e^{-E /(R T)}
\end{gathered}
$$

on domain $(0<\xi<L)$. The boundary conditions for concentration and temperature are given by

$$
\begin{aligned}
& \text { at } \xi=0, \quad \frac{\partial C}{\partial \xi}=0 ; \quad \frac{\partial T}{\partial \xi}=0 \\
& \text { at } \xi=L-D_{e} \frac{\partial C}{\partial \xi}=k\left(C-C_{s}\right) ; \quad-\lambda \frac{\partial T}{\partial \xi}=h\left(T-T_{s}\right) \\
& \text { at } \tau=0, \quad C=C_{s} ; \quad T=T_{s}
\end{aligned}
$$

Here, the boundary at $\xi=0$ is a no-flux boundary (type 2) and the boundary at $\xi=L$ is a convection boundary (type 3 ). It is convenient to create a nondimensional form of the above equations, using the following dimensionless variables:

$$
\begin{aligned}
& \theta=\frac{C-C_{s}}{C_{s}} ; \quad \phi=\frac{T-T_{s}}{T_{s}} \\
& t=\frac{D_{e} \tau}{L^{2}} ; \quad z=\frac{\xi}{L} ; \quad \mathrm{Le}=\frac{\lambda}{\rho c_{p} D_{e}} \\
& \beta=C_{s} D_{e} \frac{(-\Delta H)}{T_{s} \lambda} ; \quad \psi=\frac{L^{2} A_{0}}{D_{e}} e^{-E /\left(R T_{s}\right)} \\
& \gamma=E /\left(R T_{s}\right) ; \quad \epsilon=\frac{D_{S} T_{s}}{D_{e} C_{s}} ; \quad \omega=\frac{D_{D} C_{s}}{\lambda T_{s}}
\end{aligned}
$$

Here, $\theta$ is (unitless) concentration and $\phi$ is (unitless) temperature. Then, Eqs. (2) and (3) may be written

$$
\begin{gathered}
\frac{\partial \theta}{\partial t}=\frac{\partial^{2} \theta}{\partial z^{2}}+\epsilon \frac{\partial^{2} \phi}{\partial z^{2}}-\psi(1+\theta) \exp \left[\gamma-\frac{\gamma}{(1+\phi)}\right] \\
\frac{1}{\operatorname{Le}} \frac{\partial \phi}{\partial t}=\frac{\partial^{2} \phi}{\partial z^{2}}+\omega \frac{\partial^{2} \theta}{\partial z^{2}}+\beta \psi(1+\theta) \exp \left[\gamma-\frac{\gamma}{(1+\phi)}\right]
\end{gathered}
$$

The cross coefficient $\epsilon$ controls the induced mass fluxes that occur due to a temperature gradient without a corresponding concentration gradient. The cross coefficient $\omega$ controls the induced heat flux by chemical potential gradient of substance without temperature gradient. The induced effects controlled by $\epsilon$ and $\omega$ show the impact of thermodynamic coupling on the transient heat and mass transfer. The boundary conditions and initial conditions are given by

$$
\begin{array}{ll}
\text { at } z=0, & \frac{\partial \theta}{\partial z}=0 ; \quad \frac{\partial \phi}{\partial z}=0 \\
\text { at } z=1, & \frac{\partial \theta}{\partial z}+B_{c} \theta=0 ; \quad \frac{\partial \phi}{\partial z}+B_{T} \phi=0 \\
\text { at } t=0, & \theta=0 ; \quad \phi=0
\end{array}
$$

The purpose of this normalization is to simplify the initial conditions by setting them to zero and to make the boundary conditions homogeneous. At the $z=1$ boundary, $B_{c}=k L / D_{e}$ is the masstransfer Biot number and $B_{T}=h L / \lambda$ is thermal Biot number. We 
view the boundary condition at $z=1$ as a generalized boundary condition, because it can provide boundaries of type $1\left(B_{c} \rightarrow \infty\right.$ and $B_{T} \rightarrow \infty$ produces $\theta=\phi=0$ there) or type 2 ( $B_{c}=0$ and $B_{t}=0$ produces the zero-flux boundary) or type 3 (Biot numbers neither infinite nor zero). See Ref. [32] for further discussion of the generalized boundary condition.

\section{Semi-Analytical Source Method}

The solution method introduced in this paper treats the crossdependent terms in the above differential equations as source terms. That is, Eqs. (6) and (7) may be written

$$
\begin{gathered}
\frac{\partial \theta}{\partial t}=\frac{\partial^{2} \theta}{\partial z^{2}}+g^{1} \\
\frac{1}{\operatorname{Le}} \frac{\partial \phi}{\partial t}=\frac{\partial^{2} \phi}{\partial z^{2}}+g^{2}
\end{gathered}
$$

Quantities $g^{1}$ and $g^{2}$ are source terms, the full details of which will be discussed presently. But for the moment, the above equations appear to have the form of linear diffusion equations, driven by source terms. If this were true, and further, if the source terms were known, then classic analytic methods, such as the method of GF, would apply to this problem. The GF method would allow the problem to be recast as integrals of the product of Green's function and the (assumed known) source terms, as follows [33]:

$$
\begin{aligned}
& \theta(z, t)=\int_{t \prime=0}^{t} \int_{z \prime=0}^{L} g^{1}\left(z^{\prime}, t^{\prime}\right) G^{1}\left(z, t \mid z^{\prime}, t^{\prime}\right) d z^{\prime} d t^{\prime} \\
& \phi(z, t)=\int_{t^{\prime}=0}^{t} \int_{z \prime=0}^{L} g^{2}\left(z^{\prime}, t^{\prime}\right) G^{2}\left(z, t \mid z^{\prime}, t^{\prime}\right) d z^{\prime} d t^{\prime}
\end{aligned}
$$

The GF depends on observation location $(z, t)$ and source location $\left(z^{\prime}, t^{\prime}\right)$, while the source functions $g^{1}$ and $g^{2}$ depend only on source location $\left(z^{\prime}, t^{\prime}\right)$. The above integral statement of the solution is not formally correct, as the source terms are not actually known; however, it suggests an algorithm for a solution if the source terms can found approximately.

The source terms are given by

$$
\begin{aligned}
& g^{1}=\epsilon \frac{\partial^{2} \phi}{\partial z^{2}}-\psi(1+\theta) \exp \left[\gamma-\frac{\gamma}{(1+\phi)}\right] \\
& g^{2}=\omega \frac{\partial^{2} \theta}{\partial z^{2}}+\beta \psi(1+\theta) \exp \left[\gamma-\frac{\gamma}{(1+\phi)}\right]
\end{aligned}
$$

We treat parameters $\psi, \beta, \gamma, L e, \epsilon$, and $\omega$ as known values. The source terms depend directly on concentration $\theta$ and temperature $\phi$, both of which are functions of space and time, and they also contain a nonlinear reaction-kinetics term.

The solution algorithm based on the above integral expressions involves stepping through time, starting by constructing the first value of sources $g^{1}$ and $g^{2}$ from the initial condition. At each successive timestep, sources $g^{1}$ and $g^{2}$ will be evaluated numerically from the previous timestep, and then values of $\theta$ and $\phi$ at the next timestep will be found from the above integral description. The level of approximation for this approach depends upon the size of the timesteps and on the rate at which the source terms change over time.

3.1 Discretization Into Subintervals. As part of the solution method, time is discretized into $N$ equal-spaced intervals $\left(t_{1}, t_{2}, \ldots, t_{N}\right)$, and space is discretized into $M$ equal-sized intervals of size $\Delta z$. The source terms are approximated as piecewise constant in each time interval and in each spatial interval. The concentration and temperature functions are evaluated at the end of each time interval and in the center of each spatial interval. By this procedure, each integral in Eqs. (11) and (12) may be replaced by a sum of smaller integrals, where each smaller integral covers one subinterval in time or space, as follows:

$$
\begin{aligned}
& \theta\left(z_{i}, t_{N}\right)=\sum_{k=1}^{N} \sum_{j=1}^{M} g_{k j}^{1} \int_{t_{k-1}}^{t_{k}} \int_{z_{j}-\Delta z / 2}^{z_{j}+\Delta z / 2} G^{1}\left(z_{i}, t_{N} \mid z^{\prime}, t^{\prime}\right) d z^{\prime} d t^{\prime} \\
& \phi\left(z_{i}, t_{N}\right)=\sum_{k=1}^{N} \sum_{j=1}^{M} g_{k j}^{2} \int_{t_{k-1}}^{t_{k}} \int_{z_{j}-\Delta z / 2}^{z_{j}+\Delta z / 2} G^{2}\left(z_{i}, t_{N} \mid z^{\prime}, t^{\prime}\right) d z^{\prime} d t^{\prime}
\end{aligned}
$$

Because source terms $g^{1}$ and $g^{2}$ are piecewise constant in each subinterval, these have been moved outside the integrals. The remaining integrals over the subregions involve only Green's function. The required integrals can be tabulated beforehand in the form

$$
\Delta I^{p}\left(z_{i}, t_{N} \mid z_{j}, t_{k}\right)=\int_{t_{k-1}}^{t_{k}} \int_{z_{j}-\Delta z / 2}^{z_{j}+\Delta z / 2} G^{p}\left(z_{i}, t_{N} \mid z^{\prime}, t^{\prime}\right) d z^{\prime} d t^{\prime}
$$

where $p=1$ or 2 indicates concentration or temperature. Influence function $\Delta I^{p}$ is the response at $\left(z_{i}, t_{N}\right)$ to an internal source of unit size occurring over time interval $t_{k-1}<t<t_{k}$ and over spatial interval of size $\Delta z$ centered at location $z_{j}$. More information on how influence function $\Delta I^{p}$ is evaluated is given in the Appendix. Then the concentration and temperature may be written as

$$
\begin{aligned}
& \theta\left(z_{i}, t_{N}\right)=\sum_{k=1}^{N} \sum_{j=1}^{M} g_{k j}^{1} \Delta I^{1}\left(z_{i}, t_{N} \mid z_{j}, t_{k}\right) \\
& \phi\left(z_{i}, t_{N}\right)=\sum_{k=1}^{N} \sum_{j=1}^{M} g_{k j}^{2} \Delta I^{2}\left(z_{i}, t_{N} \mid z_{j}, t_{k}\right)
\end{aligned}
$$

3.2 Construction of Source Terms. One computational challenge in this problem is that the source terms depend on concentration $\theta$ and temperature $\phi$. In the present embodiment of the problem, this challenge is met by stepping through time and evaluating the source terms at the previous timestep, that is, when the values of $\theta$ and $\phi$ are known. Iteration can be used to improve the value of the source terms.

Another challenge is that the source terms contain spatial derivatives $\partial^{2} \theta / \partial z^{2}$ and $\partial^{2} \phi / \partial z^{2}$. Specifically, the needed spatial derivatives are given by:

$$
\begin{aligned}
& \frac{\partial^{2} \theta}{\partial z^{2}}\left(z_{i}, t_{N}\right)=\sum_{k=1}^{N} \sum_{j=1}^{M} g_{j k}^{1} \Delta S^{1}\left(z_{i}, t_{N} \mid z_{j}, t_{k}\right) \\
& \frac{\partial^{2} \phi}{\partial z^{2}}\left(z_{i}, t_{N}\right)=\sum_{k=1}^{N} \sum_{j=1}^{M} g_{j k}^{2} \Delta S^{2}\left(z_{i}, t_{N} \mid z_{j}, t_{k}\right)
\end{aligned}
$$

where

$$
\Delta S^{p}\left(z_{i}, t_{N} \mid z_{j}, t_{k}\right)=\int_{t_{k-1}}^{t_{k}} \int_{z_{j}-\Delta z / 2}^{z_{j}+\Delta z / 2} \frac{\partial^{2} G^{p}}{\partial z^{2}}\left(z_{i}, t_{N} \mid z^{\prime}, t^{\prime}\right) d z^{\prime} d t^{\prime}
$$

for $p=1$ or 2. It is important to note that because the derivative is carried out with respect to observation location $z$, the derivative bypasses the source term and falls only on Green's function. For this reason, influence function $\Delta S^{p}$ may be evaluated beforehand to high precision and stored for rapid computation. More information on evaluating influence function $\Delta S^{p}$ is given in the Appendix. 
3.3 Timestepping Solution. In this section, the timestepping solution procedure is described. Briefly, the method involves evaluating the source terms at the present time, assuming the source terms are constant over the next timestep, and evaluating the concentration and temperature using Eqs. (18) and (19) at one timestep into the future. To demonstrate the method, the calculation will be explicitly written out for the first two timesteps.

At time $t=0$, the initial conditions are $\theta=0$ and $\phi=0$ everywhere in the body. The source terms at the initial condition may be evaluated from Eqs. (13) and (14) to be

$$
\begin{gathered}
g_{1 i}^{1}=\epsilon \cdot 0-\psi(1+0) \exp \left[\gamma-\frac{\gamma}{(1+0)}\right] \\
g_{1 i}^{2}=\omega \cdot 0+\beta \psi(1+0) \exp \left[\gamma-\frac{\gamma}{(1+0)}\right]
\end{gathered}
$$

That is, initially the source terms are spatially uniform. Then, Eqs. (18) and (19) are evaluated at $N=k=1$

$$
\begin{aligned}
& \theta\left(z_{i}, t_{1}\right)=\sum_{j=1}^{M} g_{1 j}^{1} \Delta I^{1}\left(z_{i}, t_{1} \mid z_{j}, t_{1}\right) \\
& \phi\left(z_{i}, t_{1}\right)=\sum_{j=1}^{M} g_{1 j}^{2} \Delta I^{2}\left(z_{i}, t_{1} \mid z_{j}, t_{1}\right)
\end{aligned}
$$

To carry out the next timestep, the source functions for the second timestep are evaluated using concentration and temperature at time $t_{1}$

$$
\begin{aligned}
& g_{2 i}^{1}=\left.\epsilon \frac{\partial^{2} \phi}{\partial z^{2}}\right|_{\left(z_{i}, t_{1}\right)}-\psi\left(1+\theta\left(z_{i}, t_{1}\right)\right) \exp \left[\gamma-\frac{\gamma}{\left(1+\phi\left(z_{i}, t_{1}\right)\right)}\right] \\
& g_{2 i}^{2}=\left.\omega \frac{\partial^{2} \theta}{\partial z^{2}}\right|_{\left(z_{i}, t_{1}\right)}+\beta \psi\left(1+\theta\left(z_{i}, t_{1}\right)\right) \exp \left[\gamma-\frac{\gamma}{\left(1+\phi\left(z_{i}, t_{1}\right)\right)}\right]
\end{aligned}
$$

Then, Eqs. (18) and (19) are evaluated at $N=2$ and $k=1,2$

$$
\begin{aligned}
& \theta\left(z_{i}, t_{2}\right)=\sum_{j=1}^{M} g_{1 j}^{1} \Delta I^{1}\left(z_{i}, t_{2} \mid z_{j}, t_{1}\right)+\sum_{j=1}^{M} g_{2 j}^{1} \Delta I^{1}\left(z_{i}, t_{2} \mid z_{j}, t_{2}\right) \\
& \phi\left(z_{i}, t_{2}\right)=\sum_{j=1}^{M} g_{1 j}^{2} \Delta I^{2}\left(z_{i}, t_{2} \mid z_{j}, t_{1}\right)+\sum_{j=1}^{M} g_{2 j}^{2} \Delta I^{2}\left(z_{i}, t_{2} \mid z_{j}, t_{2}\right)
\end{aligned}
$$

This procedure is repeated for all successive timesteps, with three spatial sums needed at $N=3$, four spatial sums needed at $N=4$, and so on as required by the $k$-summation in Eqs. (18) and (19). This behavior arises because the summation over time index $k$ is a convolution sum. In the above description, no iterative improvement is applied to the source terms, which can be important in highly nonlinear problems. In Sec. 3.4, the incorporation of iterative improvement is described.

3.4 Iterative Improvement. Iterative improvement for the source terms at each timestep can be carried out with little additional computational cost. The reason is that the convolution sum, which is the computation-intensive part of the method, needs to be carried out only once per timestep.

A detailed description of the iteration procedure is given next. At the start of a new timestep, in preparation for iteration, the convolution sums (one each for concentration and temperature) are truncated by computing over previous timesteps only, that is, over $k=1,2, \ldots, N-1$. The truncated convolution sums do not include the effect of the present timestep. Also, the first guess for the source terms are computed using known values of concentration and temperature, from the previous timestep. Then the temperature and concentration are updated by adding the effect of the present-timestep source terms $g_{N j}^{p}$ to the truncated convolution sums as follows:

$$
\begin{aligned}
\theta\left(z_{i}, t_{N}\right) & =\sum_{k=1}^{N-1} \sum_{j=1}^{M} g_{k j}^{1} \Delta I^{1}\left(z_{i}, t_{N} \mid z_{j}, t_{k}\right)+\sum_{j=1}^{M} g_{N j}^{1} \Delta I^{1}\left(z_{i}, t_{N} \mid z_{j}, t_{N}\right) \\
\phi\left(z_{i}, t_{N}\right) & =\sum_{k=1}^{N-1} \sum_{j=1}^{M} g_{k j}^{2} \Delta I^{2}\left(z_{i}, t_{N} \mid z_{j}, t_{k}\right)+\sum_{j=1}^{M} g_{N j}^{2} \Delta I^{2}\left(z_{i}, t_{N} \mid z_{j}, t_{N}\right)
\end{aligned}
$$

In the above expressions, the double sum is the truncated convolution sum which gives the effect of previous sources, and the single sum is the effect of the present timestep sources evaluated at $t_{N}$. Note that only one matrix multiplication is required to update $\theta$ and $\phi$, and no matrix inversion is required. For the next iteration, the updated $\theta$ and $\phi$ values are used to update the source terms, and then $\theta$ and $\phi$ are themselves updated using the above expressions. Again, as sources at time $t_{N}$ are updated, the convolution sum does not have to be recomputed. Iteration stops when the relative changes in both $\theta$ and $\phi$ are sufficiently small; specifically, if $r$ is the index of iteration and $\phi_{i}^{r}$ and $\theta_{i}^{r}$ are the temperature and concentration values at spatial node $i$ after $r$ iterations, then iteration stops when

$$
\sum_{i=1}^{M}\left|\left(\phi_{i}^{r}-\phi_{i}^{r-1}\right) / \phi_{i}^{r}\right|+\sum_{i=1}^{M}\left|\left(\theta_{i}^{r}-\theta_{i}^{r-1}\right) / \theta_{i}^{r}\right|<\text { tol }
$$

When this condition is satisfied, the calculation proceeds to the next timestep. For numerical results presented in this paper, a relative change of less than tol $=0.001$ is achieved after three iterations and a relative change of less than tol $=10^{-6}$ is achieved after six iterations.

\section{Comparison With Benchmark Case}

To quantify the utility and accuracy of the method, a comparison was made with a benchmark problem for which an exact solution could be found. Consider the transient heat transfer in a finite-length fin that satisfies the following boundary value problem:

$$
\begin{aligned}
\frac{\partial T}{\partial t} & =\frac{\partial^{2} T}{\partial z^{2}}-m^{2}\left(T-T_{s}\right) ; \quad 0<z<1 ; \quad t>0 \\
\text { at } z & =0, \quad \frac{\partial T}{\partial z}=0 \\
\text { at } z & =1, \quad T=T_{0} \\
\text { at } t & =0, \quad T=T_{0}
\end{aligned}
$$

Here, $T_{s}$ is the surrounding fluid temperature, $T_{0}$ is the initial temperature, and $m$ is the dimensionless fin parameter. To apply the present method, the source term is set to

$$
g^{2}=-m^{2}\left(T-T_{s}\right)
$$

and the temperature is normalized as $\phi=T-T_{0}$. The influence functions already discussed were used to produce numerical results by taking $B_{T}=10^{10}$ which provides the type 1 boundary condition at $z=1$.

The exact solution of the above transient fin problem is found from a transformation [33] and Green's function method. The solution is 

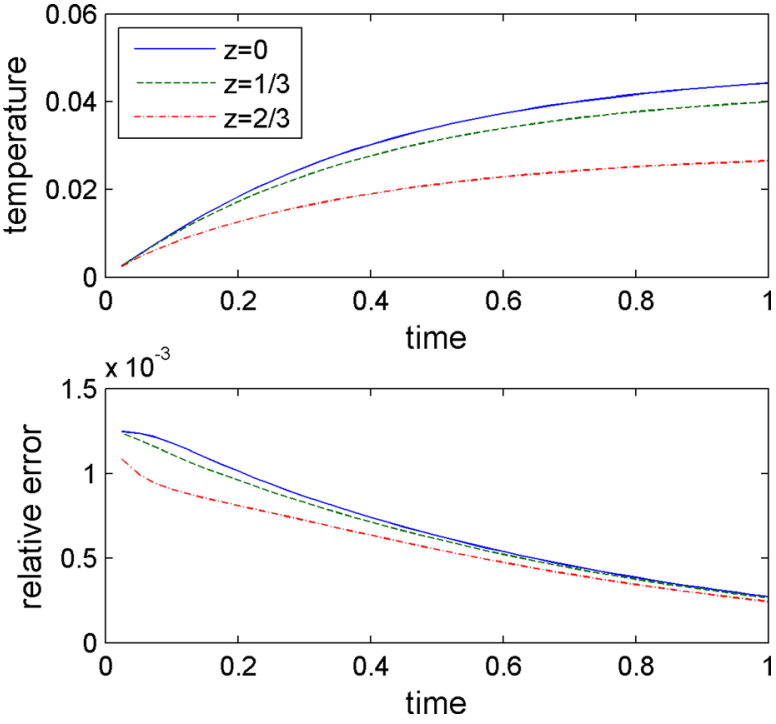

Fig. 1 (a) Temperature versus time in transient fin at three locations and (b) error between exact solution (Eq. (35)) and SAS method at three locations, for conditions $M=N=40$ and $m^{2}=0.1$

$$
\begin{aligned}
\frac{T(z, t)-T_{0}}{T_{s}-T_{0}}= & 1-\frac{\cosh z}{\cosh m} \\
& +2 \sum_{n=1}^{\infty}(-1)^{n} \cos \left(\beta_{n} z\right)\left[\frac{1}{\beta_{n}}-\frac{\beta_{n}}{\beta_{n}^{2}+m^{2}}\right] e^{-\left(\beta_{n}^{2}+m^{2}\right) t}
\end{aligned}
$$

where $\beta_{n}=(n-1 / 2) \pi$

A comparison of the exact solution with the present method is given in Fig. 1. Figure 1 $(a)$ shows the dimensionless fin temperature versus time at three locations, and Fig. 1(b) shows the relative error between the present method and the exact solution. Table 1 shows values of the relative error at $x=0$ at different values of the time, different values of the fin parameter $m^{2}$, and different values of the discretization parameters $M$ (spatial elements) and $N$ (timesteps). Location $x=0$ was chosen because it has the largest error in the body at any instant. The relative error is computed as

$$
\text { err }=\left(T_{\text {exact }}-T_{\text {SAS }}\right) / T_{\text {exact }}
$$

The purpose of Table 1 is to explore how the discretization parameters $M$ and $N$ affect the precision of the SAS method under different conditions, and also to determine the impact of iterative improvement in this benchmark case. Table 1 shows that the relative error is everywhere less than 0.0012 without iteration and is smaller when iteration is added. The error decreases as the number of spatial elements $M$ increases, decreases as the number of timesteps $N$ increases, is somewhat sensitive to time $t_{\max }$, and is somewhat sensitive to fin parameter $\mathrm{m}^{2}$. A unifying theme is that the error is larger when temperature changes rapidly (small time, small fin parameter) and is smaller when the temperature is steady or nearly steady (large time, large fin parameter).

\section{Results}

In this section, the results are given for the reaction-diffusion problem for a specific case to represent synthesis of vinyl chloride with parameter values taken from Demirel [31]. The parameter values used here are given in Table 2. Note that $B_{T}=B_{c}=10^{10}$, a floating-point version of infinity, produces a homogeneous type 1 boundary condition at $z=1$, that is, $\phi=\theta=0$ there. Although other boundary conditions (type 2 or 3 ) could be explored with other values of $B_{T}$ and $B_{c}$, we present a few results with one boundary condition as a brief demonstration of the SAS method. All of the SAS-method results presented in this section have been calculated with iterative improvement, which is needed to address the inherently nonlinear behavior in the chemical reaction term, that is, term $e^{-E /(R T)}$ in Eqs. (2) and (3).

Numerical results for the reaction-diffusion problem were checked in two ways. First, a FD code was written using finite volume method of Patankar [34], and the results were compared with the present method. Some numerical values for this comparison are given in Table 3 for the SAS method, the FD method, and

Table 1 Verification of SAS method by comparison with an exact fin solution, Eq. (35). Each row gives the relative error for SAS

\begin{tabular}{|c|c|c|c|c|c|c|}
\hline$t$ & $m^{2}$ & M & $\mathrm{N}$ & Error, no iteration & Error with iteration & Error change \\
\hline 0.2 & 0.001 & 10 & 10 & 0.000008519823 & 0.000008122093 & 0.046682895 \\
\hline 0.2 & 0.1 & 10 & 10 & 0.000844178999 & 0.000802962581 & 0.048824264 \\
\hline 0.2 & 0.001 & 40 & 10 & 0.000008473749 & 0.000008204894 & 0.031727987 \\
\hline 0.2 & 0.1 & 40 & 10 & 0.000839578099 & 0.000811178586 & 0.033825934 \\
\hline 1.0 & 0.001 & 10 & 10 & 0.000013156311 & 0.000010575162 & 0.196190938 \\
\hline 1.0 & 0.1 & 10 & 10 & 0.001231825174 & 0.000987102654 & 0.198666601 \\
\hline 1.0 & 0.001 & 40 & 10 & 0.000012879672 & 0.000010888886 & 0.154568067 \\
\hline 1.0 & 0.1 & 40 & 10 & 0.001204548950 & 0.001017670195 & 0.155144177 \\
\hline 0.2 & 0.001 & 10 & 40 & 0.000002156944 & 0.000002003159 & 0.071297632 \\
\hline 0.2 & 0.1 & 10 & 40 & 0.000213568274 & 0.000198162267 & 0.072136215 \\
\hline 0.2 & 0.001 & 40 & 40 & 0.000002097093 & 0.000002072203 & 0.011868811 \\
\hline 0.2 & 0.1 & 40 & 40 & 0.000207608332 & 0.000205027107 & 0.012433147 \\
\hline 1.0 & 0.001 & 10 & 40 & 0.000003329616 & 0.000002575192 & 0.226579882 \\
\hline 1.0 & 0.1 & 10 & 40 & 0.000312698854 & 0.000239309527 & 0.234696501 \\
\hline 1.0 & 0.001 & 40 & 40 & 0.000003037638 & 0.000002876399 & 0.053080387 \\
\hline 1.0 & 0.1 & 40 & 40 & 0.000284060776 & 0.000268766415 & 0.053841862 \\
\hline 0.2 & 0.001 & 10 & 160 & 0.000000589273 & 0.000000450743 & 0.235086284 \\
\hline 0.2 & 0.1 & 10 & 160 & 0.000058361167 & 0.000044574467 & 0.236230711 \\
\hline 0.2 & 0.001 & 40 & 160 & 0.000000525984 & 0.000000516331 & 0.018352269 \\
\hline 0.2 & 0.1 & 40 & 160 & 0.000052062587 & 0.000051099301 & 0.018502461 \\
\hline 1.0 & 0.001 & 10 & 160 & 0.000001058215 & 0.000000417535 & 0.605434623 \\
\hline 1.0 & 0.1 & 10 & 160 & 0.000100337130 & 0.000037628041 & 0.624983882 \\
\hline 1.0 & 0.001 & 40 & 160 & 0.000000762691 & 0.000000715365 & 0.062051342 \\
\hline 1.0 & 0.1 & 40 & 160 & 0.000071384407 & 0.000066785421 & 0.064425639 \\
\hline
\end{tabular}
method twice, once with and without iterative improvement, at several values of the time $t$, fin parameter $m^{2}$, spatial discretization $M$ and time discretization $N$. The fractional change between the two error columns is also listed. 
Table 2 Parameters for numerical results

\begin{tabular}{lc}
\hline \hline Parameter & Value \\
\hline$\psi$ & 0.27 \\
$\beta$ & 0.25 \\
$L e$ & 0.10 \\
$\gamma$ & 6.50 \\
$\epsilon$ & (varies) \\
$\omega$ & (varies) \\
$B_{T}$ & $10^{10}$ \\
$B_{c}$ & $10^{10}$ \\
\hline \hline
\end{tabular}

the fractional error between them. The values in this table were computed for specific case $\epsilon=\omega=0$ for verification purposes. That is, the cross-dependency terms associated with the Dufour and Soret effects are not present in Table 3. The SAS methods given were carried out with discretization at 20 timesteps and 20 spatial nodes, and the finite difference method was carried out with discretization at 1000 timesteps and 100 spatial nodes. The results in Table 3 show that the two solution methods agree very closely, with fractional error everywhere less than 0.0001 in concentration and less than 0.000028 in temperature. The error in concentration between the two methods decreases slightly with time and location, but the difference in temperature has trends that are less clear. This comparison demonstrates verification of the SAS method because it agrees within one part in ten thousand with an independent numerical method carried out with many more timesteps and more spatial nodes.

A second method of checking the results was a comparison with a commercial finite element software COMSOL MULTIPHYSICS. Some numerical values at dimensionless time $t=1.0$ are given in Table 4. The results shown in Table 4 are carried out with $\epsilon=\omega=0.001$, that is, for nonzero cross dependency of the concentration and temperature associated the Dufour effect and the Soret effect, at levels suggested by Demirel [30]. For this comparison, the present method was carried out with discretization values $N=M=20$ and the comsol code used 100 quadratic elements and 1000 timesteps. Table 3 shows values for concentration $(\theta+$ $1)=C / C_{s}$ and temperature $(\phi+1)=T / T_{s}$ and the relative error between the two methods. The relative error is defined in a
Table 4 Temperature $(\phi+1)=T / T_{s}$ and concentration $(\theta+1)$ $=C / C_{s}$ from the SAS method $(M=20, N=20)$ and the error when these values are compared with commercial software сомsol $(M=100, N=1000)$ at time $t=1.0$. Here, cross-dependency effects are included by $\epsilon=\omega=0.001$.

\begin{tabular}{lcccc}
\hline \hline$z$ & $T / T_{s}$ & $C / C_{s}$ & $T / T_{s}-$ error & $C / C_{s}$-error \\
\hline 0.15 & 1.00626256 & 0.88655192 & -0.00333566 & -0.003967174 \\
0.25 & 1.00619887 & 0.89102061 & -0.00349582 & -0.003926474 \\
0.35 & 1.00607647 & 0.89775375 & -0.00372704 & -0.003866266 \\
0.45 & 1.00586078 & 0.90678666 & -0.00400572 & -0.003787671 \\
0.55 & 1.00550249 & 0.91816498 & -0.00429279 & -0.003691874 \\
0.65 & 1.00493611 & 0.93194337 & -0.00454452 & -0.003580322 \\
0.75 & 1.00407960 & 0.94818374 & -0.00471966 & -0.003454612 \\
0.85 & 1.00283550 & 0.96695301 & -0.00479614 & -0.003316126 \\
0.95 & 1.00109393 & 0.98832045 & -0.00477611 & -0.003166669 \\
\hline \hline
\end{tabular}

manner similar to Eq. (36). The results agree within $0.5 \%$ for concentration and within $0.4 \%$ for temperature, that is, the present method agrees with an independent solution of the problem.

Figure 2 shows the spatial distribution of the solution at dimensionless times $t=0.0,0.25,0.5,1.0$. Figures $2(a)$ and $2(b)$ show concentration $(\theta+1)=C / C_{s}$ and temperature $(\phi+1)=T / T_{s}$, Figs. 2(c) and 2(d) show source terms $g^{1}$ and $g^{2}$ under the same conditions. Note that the $C / C_{s}$ curves in Fig. 2(a) have a curved shape as time evolves, but that the $T / T_{s}$ curves have a flat region at small $z$ at early time. This shape difference indicates that the concentration quickly reaches a quasi-steady behavior, but that temperature is far from steady. This is a consequence of the value $\mathrm{Le}=0.1$ which causes temperature to evolve more slowly than concentration.

Figure 3 shows the same information at later times, $t=0.0,0.75,1.5,3.0$. The concentration $\left(C / C_{s}\right.$ in Fig. $\left.3(a)\right)$ is approaching steady-state (the curves are close together) while temperature $\left(T / T_{s}\right)$ is still changing. However, the temperature curves are no longer flat near $z=0$. The shape of the source terms in Figs. 3(c) and 3(d) contain inflection points at time $t=3.0$, in contrast to earlier time when the source terms are monotonic. The inflection in the shape of the source terms is caused by the time evolution of the source terms - the source values near $x=0$ first move away from the initial values during time $t=0$ to about

Table 3 Temperature $(\phi+1)=T / T_{s}$ and concentration $(\theta+1)=C / C_{s}$ for the reaction-diffusion problem by the SAS method $(M=20$, $N=20$ ) and by a finite difference code (timestep 0.001 , spatial step 0.01 ). The fractional error between them is also listed, as a verification of the SAS method. The parameters used in the calculation are given in Table 2, except here $\epsilon=\omega=0$, that is, the crossdependency terms are zero.

\begin{tabular}{|c|c|c|c|c|c|c|c|}
\hline \multirow[b]{2}{*}{$t$} & \multirow[b]{2}{*}{$x$} & \multicolumn{2}{|c|}{ SAS Method } & \multicolumn{2}{|c|}{ FD Method } & \multicolumn{2}{|c|}{ Fractional error } \\
\hline & & $T / T_{s}$ & $C / C_{s}$ & $T / T_{s}$ & $C / C_{s}$ & $T / T_{s}$ & $C / C_{s}$ \\
\hline \multirow[t]{9}{*}{0.50} & 0.15 & 1.00323275 & 0.91136032 & 1.00323089 & 0.91144578 & 0.00000185 & -0.00009377 \\
\hline & 0.25 & 1.00323060 & 0.91460112 & 1.00322877 & 0.91468156 & 0.00000182 & -0.00008795 \\
\hline & 0.35 & 1.00321890 & 0.91952802 & 1.00321710 & 0.91960111 & 0.00000179 & $-0.0000794 \mathrm{C}$ \\
\hline & 0.45 & 1.00318236 & 0.92621848 & 1.00318067 & 0.92628210 & 0.00000168 & -0.0000686 \\
\hline & 0.55 & 1.00309195 & 0.93477341 & 1.00309049 & 0.93482566 & 0.00000146 & -0.00005590 \\
\hline & 0.65 & 1.00289773 & 0.94531493 & 1.00289674 & 0.94535425 & 0.00000099 & -0.0000415 \\
\hline & 0.75 & 1.00252206 & 0.95798348 & 1.00252189 & 0.95800861 & 0.00000017 & -0.00002623 \\
\hline & 0.85 & 1.00185568 & 0.97293399 & 1.00185669 & 0.97294405 & -0.00000101 & -0.00001034 \\
\hline & 0.95 & 1.00075925 & 0.99033115 & 1.00076174 & 0.99032561 & -0.00000249 & 0.0000055 \\
\hline \multirow[t]{9}{*}{1.00} & 0.15 & 1.00627105 & 0.88649332 & 1.00626905 & 0.88652411 & 0.00000199 & -0.00003473 \\
\hline & 0.25 & 1.00620686 & 0.89096515 & 1.00620496 & 0.89099369 & 0.00000189 & -0.00003203 \\
\hline & 0.35 & 1.00608375 & 0.89770285 & 1.00608204 & 0.89772810 & 0.00000170 & -0.00002813 \\
\hline & 0.45 & 1.00586717 & 0.90674157 & 1.00586577 & 0.90676257 & 0.00000139 & -0.00002316 \\
\hline & 0.55 & 1.00550785 & 0.91812675 & 1.00550691 & 0.91814265 & 0.00000093 & -0.00001732 \\
\hline & 0.65 & 1.00494035 & 0.93191283 & 1.00494007 & 0.93192294 & 0.00000028 & -0.00001085 \\
\hline & 0.75 & 1.00408266 & 0.94816150 & 1.00408323 & 0.94816526 & -0.00000057 & -0.00000397 \\
\hline & 0.85 & 1.00283735 & 0.96693951 & 1.00283894 & 0.96693652 & -0.00000159 & 0.00000305 \\
\hline & 0.95 & 1.00109455 & 0.98831592 & 1.00109728 & 0.98830597 & -0.00000273 & 0.00001007 \\
\hline
\end{tabular}



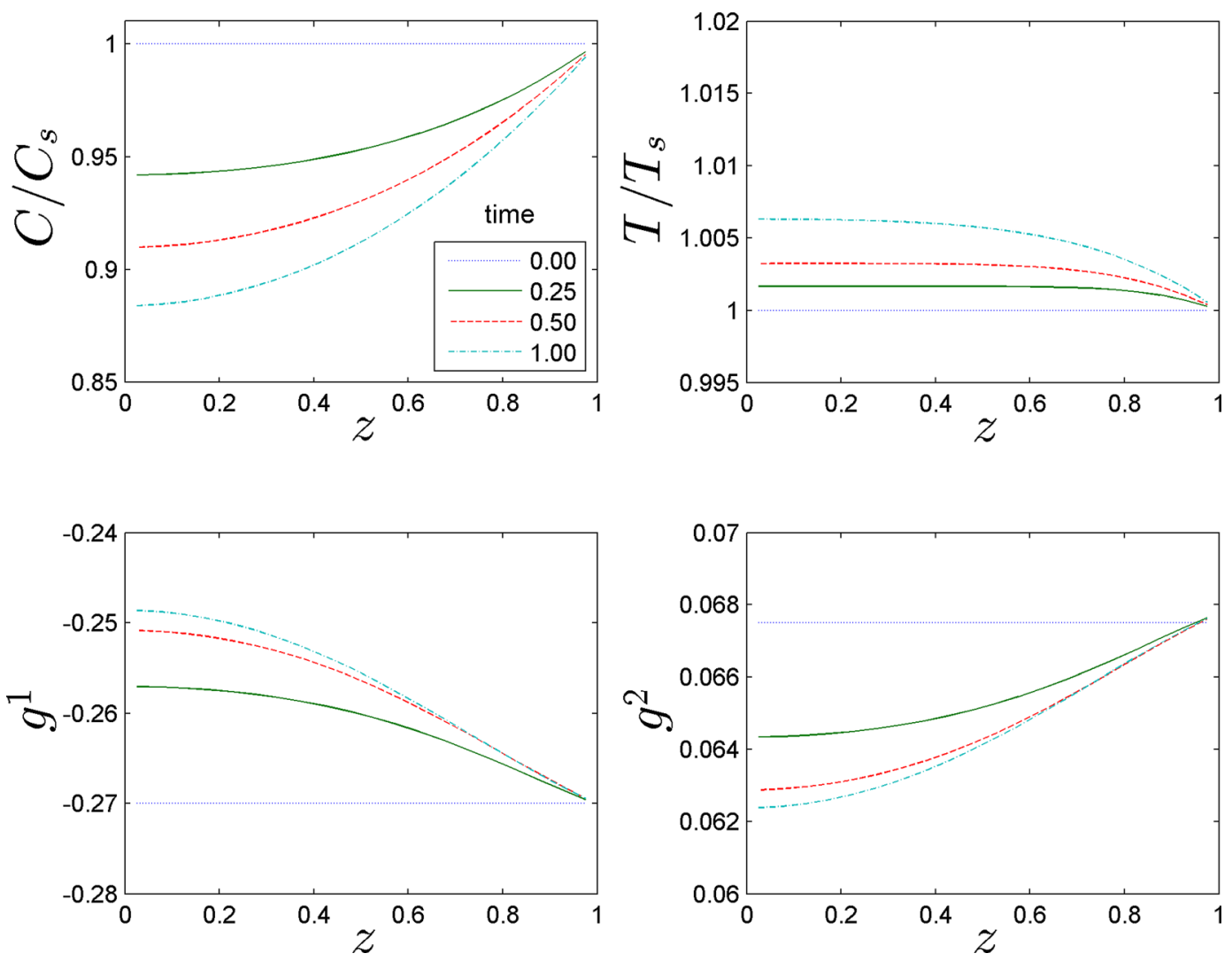

Fig. 2 Results from SAS method at dimensionless times $t=0.0,0.25,0.5,1.0:(a)$ concentration $C / C_{s},(b)$ temperature $T / T_{s},(c)$ source $g^{1}$, and (d) source $g^{2}$
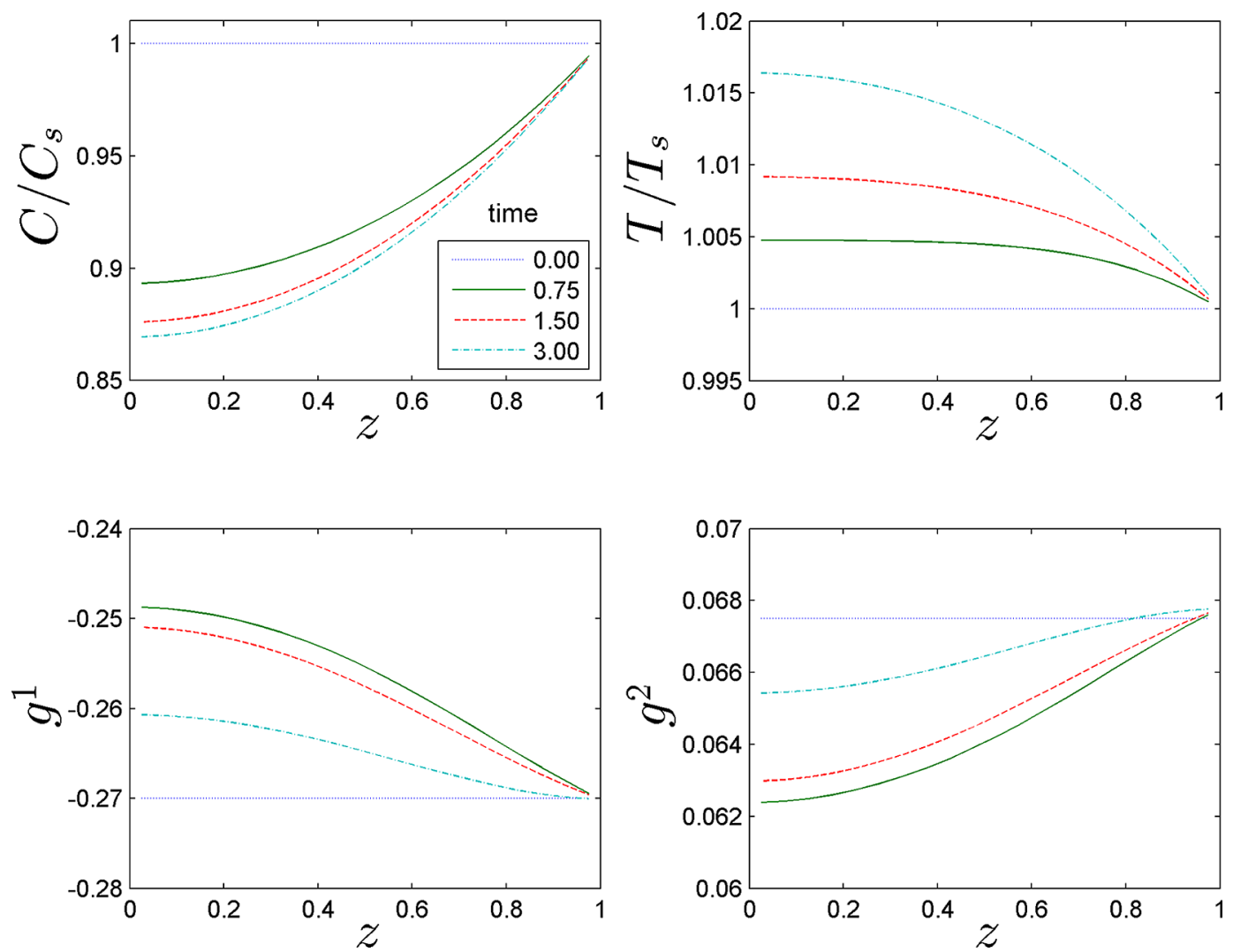

Fig. 3 Results from SAS method at dimensionless times $t=0.0,0.75,1.5,3.0$ : (a) concentration $C / C_{s},(b)$ temperature $T / T_{s},(c)$ source $g^{1}$, and $(d)$ source $g^{2}$ 
$t=0.8$, and then relax gradually back toward the initial values thereafter. That the shape of the source terms plotted in Figs. 2 and 3 is nonuniform in space is due almost entirely to the reaction term in the differential equation (that is, the term multiplied by $(1+\theta) e^{-E /(R T)}$ in Eqs. (6) and (7)). There is a very small impact from the Dufour effect and Soret effect, as discussed below.

A central motivation of this paper is to provide a solution method that can treat reaction-diffusion problems that include the cross dependency effects of Dufour and Soret, the size of which is determined by dimensionless parameters $\epsilon$ and $\omega$. Numerical results already presented provide an opportunity to see the size of the cross dependency effects on the temperature and concentration. Recall that Table 3 contains SAS method results for $\epsilon=\omega=$ 0 and Table 4 contains SAS method results with $\epsilon=\omega=0.001$. An examination of numerical values at $t=1.0$ for the SAS method results in Tables 3 and 4 shows that the results differ by less than 0.0004 for concentration and about 0.000009 for temperature at $x=0.15$; the difference is less at larger $x$ values. Results for this comparison (that is, results with and without cross dependencies) are not plotted because the difference is too small to be visible on the scale used for Figs. 2 and 3.

An informed application of a numerical method requires identifying what values of the discretization parameters provide sufficiently accurate results. Based on the comparison with COMSOL, $N=M=20$ appear adequate for results accurate within $0.5 \%$. A further exploration of a range of discretization parameters was carried out, and the results were compared to "best" results at $N=160$ and $M=162$ at a specific point in time and space $(z=1 / 12, t=0.5)$. Values of relative error in concentration $(\theta)$ in the form $\left(\theta-\theta_{\text {best }}\right) / \theta_{\text {best }}$ are plotted in Fig. 4 versus the number of timesteps. The relative error in temperature ( $\phi$, not shown) has similar trends. Figure 4 shows that discretization $N=6, M=6$ is sufficient for results accurate within $0.4 \%$. Discretization with $N \approx 25$ timesteps provides results within $0.1 \%$. These discretization values are extremely small compared to fully numeric methods, for example, COMSOL required $M=100 N=1000$ for comparably precise results. The shape of the curves in Fig. 4 is important, as the curves change rapidly for $N<25$ and changes more slowly for $N>25$. That is, adding timesteps beyond $N=25$ has a very small impact on the results. The new method gives high precision with a very coarse mesh, suggesting that the method will be computationally parsimonious, especially for repetitive calculations needed for inverse problems and process-

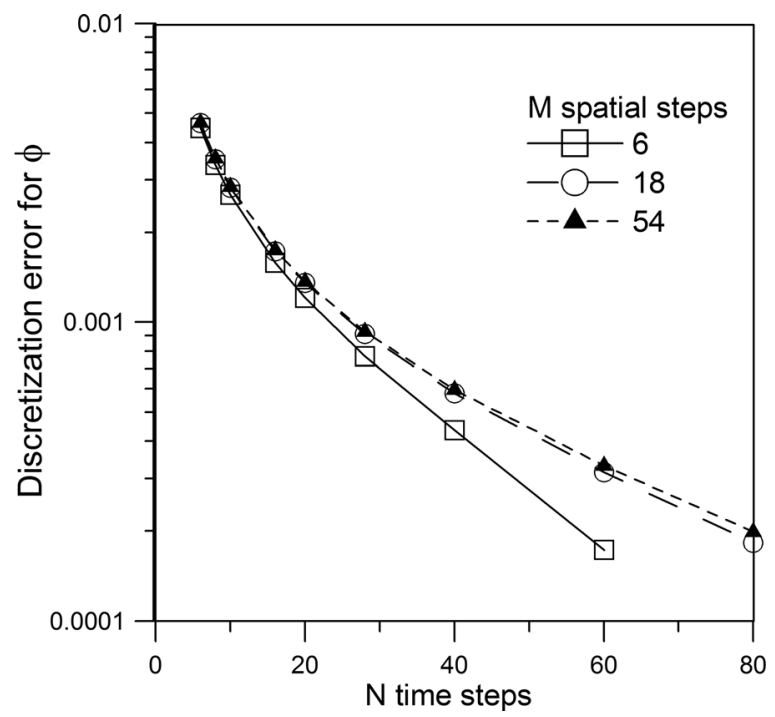

Fig. 4 Relative error in concentration $(\theta)$ at $t=0.5$ as discretization parameters $N$ and $M$ are varied. A very coarse mesh (18 elements, 6 timesteps) is adequate for $0.4 \%$ precision. monitoring problems, when the influence functions can be computed once and stored for re-use.

Some finer points of Fig. 4 will be discussed next. Values of $M=6,18,54$ (tripled each time) were chosen so that the $z=1$ / 12 observation location was conveniently located at the center of a spatial element. The $M=6$ curve is limited to few timesteps (small $N$ ) because at higher $N$ the error becomes negative and could not be included in a semi-log plot. Also, the error appears to be slightly smaller for lowering $M$ alone, an unexpected effect which is partly explained by the change of sign in the error when $M$ is small. That is, at small $M$, the values are biased to one side (positive error) for few timesteps $(\operatorname{small} N$ ) and are biased to the other side (negative error) for more timesteps $(\operatorname{larger} N$ ). Although this unexpected effect at small $M$ is a point for future investigation, this effect fades away for the range of values $(M>18)$ used for numerical results presented in this paper. Further, the size of this unexpected variation in error with $M$ is vanishingly small compared with that for fully numeric methods. Again, the takeaway message from Fig. 4 is that the error is sensitive to the number of timesteps and insensitive to the number of spatial elements.

\section{Discussion}

A full exploration of this reaction-diffusion problem could include the sensitivity of the results to variations in the input values. Such an exploration has not been included because the operating conditions presented here are located in the vicinity of equilibrium where the behavior is nearly linear. Evidence for this viewpoint is visible in the source terms plotted in Figs. 2 and 3 which are spatially uniform at $t=0$ and then evolve into nonuniform shapes that vary at most $9 \%$ from the initial values. In this near-linear regime, small variations in the operating conditions will produce only small variations in the output results. Future work will include exploration of strongly nonlinear regimes along with appropriate sensitivity studies.

Next, the scope of additional problems to which the SAS method may be applied is discussed. In the present paper, the SAS method is based on fundamental solutions constructed from exact Green's functions. For an exact analytical solution to exist, one limitation is that the body shape must be aligned with the coordinate system so that boundaries are specified by a constant value of one coordinate; that is, it must be an orthogonal body. Generally this means that the body surfaces must be simple shapes such as planes, cylinders, or spheres. For such body shapes, the exact Green's functions are available for a variety of boundary conditions $[35,36]$. Although exact Green's functions are desirable for their precision and computational efficiency, they are not the only tool for constructing influence functions. Galerkin-based Green's functions have been demonstrated for non-orthogonal bodies and heterogeneous bodies; see for example Ref. [37].

Another limitation for the SAS method is that the boundary conditions and the differential equation (diffusion equation) must be linear. Linearity is required for superposition, the principle that adding solutions produces new solutions, which is an essential element of the SAS method. Linear boundary conditions are those in which the diffusion variable (concentration or temperature) appear only to the first power. Linear boundary conditions include types 1,2 , or 3 as discussed in this paper. A counter-example in heat transfer is the radiation boundary condition, in which temperature appears to the fourth power. For the differential equation to be linear, the material properties in the differential equation must not be functions of the temperature (or concentration in the case of mass transfer). It is important to note, however, that the limitation of linearity may be surmountable, by the fact that the SAS method as presented here uses iteration to deal with the nonlinear reactive-source terms in Eqs. (2) and (3). From this example, there is reason to suppose that some level of nonlinear effects in the boundaries and in the material properties could also be treated through iteration. 


\section{Summary and Conclusions}

In this paper, the semi-analytical source method is introduced for a reaction-diffusion problem with thermodynamic coupling between concentration and temperature. Potential applications include microreactors and microfuel cells. The method is based on exact solutions of the diffusion equation where the crossdependent terms are treated as source terms which are evaluated numerically. Nonlinear effects inside the body may be treated with iterative improvement at small additional computational cost. The new method provides precise results as evidenced by comparison with fully numeric solutions. A very coarse grid is sufficient for precise values (six spatial elements provides accuracy within $0.4 \%$ ), suggesting that the new method has great potential for saving computer time compared with fully numeric methods, especially for repetitive calculations. Although the method was demonstrated with a single geometry, other geometries and other boundary conditions may be treated using appropriate exact solutions which are widely available. Future work will extend the method to other boundary conditions, other geometries, and additional mass-diffusion constituents.

\section{Acknowledgment}

This work was supported by the University of Nebraska Foundation through the Global Faculty Associates program.

\section{Nomenclature}

$a=$ heated region (m), Eqs. (A1) and (A8)

$A_{0}=$ frequency factor $(1 / \mathrm{s})$

$B_{c}=k L / D_{e}$, mass-transfer Biot number

$B_{T}=h L / \lambda$, thermal Biot number

$C=$ concentration $\left(\mathrm{kmol} / \mathrm{m}^{3}\right)$

$c_{p}=$ specific heat $(\mathrm{J} / \mathrm{kg} / \mathrm{K})$

$D_{D}=$ coefficient related to Dufour effect $\left(\mathrm{m}^{2} \mathrm{~J} /(\mathrm{kmol} \mathrm{s})\right)$

$D_{e}=$ diffusivity $\left(\mathrm{m}^{2} / \mathrm{s}\right)$

$D_{S}=$ coefficient related to Soret effect $(\mathrm{kmol} /(\mathrm{m} \mathrm{s} \mathrm{K}))$

$E=$ activation energy $(\mathrm{kJ} / \mathrm{kmol})$

$g^{p}=$ source term, Eqs. (11) and (12)

$G^{p}=$ Green's function, $p=1,2$

$h=$ heat transfer coefficient $\left(\mathrm{W} /\left(\mathrm{m}^{2} \mathrm{~K}\right)\right)$

$J=\operatorname{mass}$ flux $\left(\mathrm{kmol} /\left(\mathrm{m}^{2} \mathrm{~s}\right)\right)$

$J_{q}=$ heat flux $\left(\mathrm{W} / \mathrm{m}^{2}\right)$

$k=$ mass transfer coefficient $(\mathrm{m} / \mathrm{s})$

$L=$ domain thickness (m)

$L_{e}=$ Lewis number, Eq. (5)

$M=$ number of spatial elements

$m^{2}=$ fin parameter, Eq. (32)

$N=$ number of timesteps

$R=$ gas constant $(\mathrm{kJ} /(\mathrm{kmol} \mathrm{K}))$

$t=$ time (unitless), Eq. (5)

$T=$ temperature $(\mathrm{K})$

$z=$ coordinate (unitless), Eq. (5)

$\Delta H=$ heat of reaction $(\mathrm{kJ} / \mathrm{kmol})$

$\Delta I=$ influence function, Eq. (17)

$\Delta S=$ influence function, Eq. (22)

\section{Greek Symbols}

$$
\begin{aligned}
\beta & =\text { defined in Eq. }(5) \\
\beta_{n} & =\text { eigenvalue } \\
\gamma & =\text { defined in Eq. }(5) \\
\delta & =\text { Dirac delta in Eq. (A1) } \\
\epsilon & =\text { defined in Eq. }(5) \\
\theta & =\text { concentration }\left(C-C_{s}\right) / C_{s} \\
\xi & =\text { spatial coordinate }(\mathrm{m}) \\
\rho & =\text { density }\left(\mathrm{kg} / \mathrm{m}^{3}\right) \\
\lambda & =\text { thermal conductivity }(\mathrm{W} / \mathrm{m} / \mathrm{K}) \\
\tau & =\text { time }(\mathrm{s}) \\
\phi & =\text { temperature }\left(T-T_{s}\right) / T_{s}
\end{aligned}
$$

$\psi=$ defined in Eq. (5)

$\omega=$ defined in Eq. (5)

\section{Subscripts}

$$
\begin{aligned}
i & =\text { observation location } \\
j & =\text { heating location } \\
k & =\text { heating time } \\
N & =\text { observation time } \\
s & =\text { ambient value } \\
\text { SAS } & =\text { semi-analytic source method } \\
0 & =\text { initial value, Eq. }(33)
\end{aligned}
$$

\section{Appendix: Influence Functions}

In this appendix, the influence functions $\Delta I^{p}$ and $\Delta S^{p}$ are constructed using the method of GF. The GF for diffusion associated with Eq. (9) and (10) are defined by the following auxiliary problem:

$$
\begin{aligned}
& \frac{1}{L_{e}^{p}} \frac{\partial G^{p}}{\partial t}=\frac{\partial^{2} G^{p}}{\partial z^{2}}+\frac{1}{L_{e}^{p}} \delta\left(z-z^{\prime}\right) \delta\left(t-t^{\prime}\right) ; \quad 0<z<1 ; \quad t>t^{\prime} \\
& \text { at } z=0, \quad \frac{\partial G^{p}}{\partial z}=0 \\
& \text { at } z=1, \quad \frac{\partial G^{p}}{\partial z}+B_{p} G^{p}=0 \\
& \text { at } t<t^{\prime}, \quad G^{p}=0
\end{aligned}
$$

The boundary conditions for $G^{p}$ are of the same type as the original problem, that is, type 2 at $z=0$ and the generalized condition at $z=1$ where $B_{p}$ is the Biot number for concentration or temperature as appropriate. Using the heat conduction number system, this geometry is denoted X23 [33]. The unitless GF is given by [33]

$G_{X 23}^{p}\left(z, t \mid z^{\prime}, t^{\prime}\right)=2 \sum_{n=1}^{\infty} \cos \left(\beta_{n} z\right) \cos \left(\beta_{n} z^{\prime}\right) \frac{\beta_{n}^{2}+B_{p}^{2}}{\beta_{n}^{2}+B_{p}^{2}+B_{p}} e^{-\beta_{n}^{2} L_{e}^{p}(t-t \prime)}$

where $\beta_{n}$ are roots of the eigencondition $\beta_{n} \tan \beta_{n}=B_{p}$ and where coefficient $L_{\rho}^{p}$ modifies the time variable. If different boundary conditions at $x=0$ were of interest for this problem, the appropriate GF is available with generalized boundary conditions at both boundaries [36] and the solution may be developed in a similar fashion.

Function $\Delta I^{p}$ : Influence function $\Delta I^{p}$ is defined by

$$
\Delta I^{p}\left(z_{i}, t_{N} \mid z_{j}, t_{k}\right)=\int_{t_{k-1}}^{t_{k}} \int_{z_{j}-\Delta z / 2}^{z_{j}+\Delta z / 2} G^{p}\left(z_{i}, t_{N} \mid z^{\prime}, t^{\prime}\right) d z^{\prime} d t^{\prime}
$$

To facilitate numerical computation, the above function can be constructed from a simpler function defined by

$$
I^{p}\left(z_{i}, t_{N} \mid a, t_{k}\right)=\int_{0}^{t_{k}} \int_{0}^{a} G^{p}\left(z_{i}, t_{N} \mid z^{\prime}, t^{\prime}\right) d z^{\prime} d t^{\prime}
$$

Using the above function $I^{p}$, four values can be superposed to construct function $\Delta I^{p}$, as follows:

$$
\begin{aligned}
\Delta I^{p}\left(z_{i}, t_{N} \mid z_{j}, t_{k}\right) & \\
= & \left\{I^{p}\left(z_{i}, t_{N} \mid z_{j}+\frac{\Delta z}{2}, t_{k}\right)-I^{p}\left(z_{i}, t_{N} \mid z_{j}+\frac{\Delta z}{2}, t_{k-1}\right)\right\} \\
& -\left\{I^{p}\left(z_{i}, t_{N} \mid z_{j}-\frac{\Delta z}{2}, t_{k}\right)-I^{p}\left(z_{i}, t_{N} \mid z_{j}-\frac{\Delta z}{2}, t_{k-1}\right)\right\}
\end{aligned}
$$


Function $\Delta S^{p}$ : Influence function $\Delta S^{p}$ is needed for the spatial derivative of the concentration and temperature which appear in the source term. Function $\Delta S^{p}$ is defined by

$$
\Delta S^{p}\left(z_{i}, t_{N} \mid z_{j}, t_{k}\right)=\left.\int_{t_{k-1}}^{t_{k}} \int_{z_{j}-\Delta z / 2}^{z_{j}+\Delta z / 2} \frac{\partial^{2} G^{p}}{\partial z^{2}}\right|_{z=z_{i}} d z^{\prime} d t^{\prime}
$$

As the spatial derivative falls only on the observation location $z$ and not on integration variable $z^{\prime}$, function $\Delta S^{p}$ may be constructed by taking the spatial derivative of function $\Delta I^{p}$. Then function $\Delta S^{p}$ is given by

$$
\begin{aligned}
\Delta S^{p}\left(z_{i}, t_{N} \mid z_{j}, t_{k}\right) \\
=\left\{S^{p}\left(z_{i}, t_{N} \mid z_{j}+\frac{\Delta z}{2}, t_{k}\right)-S^{p}\left(z_{i}, t_{N} \mid z_{j}+\frac{\Delta z}{2}, t_{k-1}\right)\right\} \\
\quad-\left\{S^{p}\left(z_{i}, t_{N} \mid z_{j}-\frac{\Delta z}{2}, t_{k}\right)-S^{p}\left(z_{i}, t_{N} \mid z_{j}-\frac{\Delta z}{2}, t_{k-1}\right)\right\}
\end{aligned}
$$

where

$$
S^{p}\left(z_{i}, t_{N} \mid a, t_{k}\right)=\left.\int_{0}^{t_{k}} \int_{0}^{a} \frac{\partial^{2} G^{p}}{\partial z^{2}}\right|_{z=z_{i}} d z^{\prime} d t^{\prime}
$$

\section{References}

[1] Froment, G. F., and Bischoff, K. B., 1979, Chemical Reactor Analysis and Design, Wiley, New York, pp. 202-204.

[2] Heinrich, R., and Schuster, S., 1998, "The Modeling of Metabolic Systems Structure, Control and Optimality," BioSystems, 47(1-2), pp. 61-77.

[3] Burghardt, A., and Berezowski, M., 2003, "Periodic Solutions in a Porous Catalyst Pellet-Homoclinic Orbits," Chem. Eng. Sci., 58(12), pp. $2657-2670$.

[4] Gas, P., Girardeaux, C., Mangelinck, D., and Portavoce, A., 2003, "Reaction and Diffusion at Interfaces of Micro- and Nanostructured Materials," Mater. Sci. Eng. B, 101(1-3), pp. 43-48.

[5] Demirel, Y., and Sandler, S. I., 2004, "Nonequilibrium Thermodynamics in Engineering and Science," J. Phys. Chem. B, 108(1), pp. 31-43.

[6] Tevatia, R., Demirel, Y., and Blum, P., 2014, "Influence of Subenvironmental Conditions and Thermodynamic Coupling on a Simple Reaction-Transport Process in Biochemical Systems," Ind. Eng. Chem. Res., 53(18), pp. 7637-7647.

[7] Demirel, Y., and Sandler, S. I., 2001, "Linear Nonequilibrium Thermodynamics Theory for Coupled," Int. J. Heat Mass Transfer, 44(13), pp. 2439-2451.

[8] Rodrigo, M., and Mimura, M., 2001, "Exact Solutions of Reaction-Diffusion Systems and Nonlinear Wave Equations," Jpn. J. Ind. Appl. Math., 18, pp. 657-696.

[9] Turing, A., 1952, "The Chemical Basis of Morphogenesis," Philos. Trans. R. Soc. B, 237(641), pp. 37-72.

[10] Serna, H., Munuzun, A. P., and Barragan, D., 2017, "Thermodynamic and Morphological Characterization of Turing Patterns in Non-Isothermal ReactionDiffusion Systems," Phys. Chem. Chem. Phys., 19(22), pp. 14401-14411.

[11] Lobanova, E. S., Shnol, E. E., and Ataullakhanov, F. I., 2004, "Complex Dynamics of the Formation of Spatially Localized Standing Structures in the Vicinity of Saddle-Node Bifurcations of Waves in the Reaction-Diffusion Model of Blood Clotting," Phys. Rev. E, 70(3), p. 032903.

[12] Berezhkovski, A. M., Coppey, M., and Shvartsman, S. Y., 2009, "Signalling Gradients in Cascades of Two-State Reaction Diffusion Systems," PNAS, 106(4), pp. 1087-1092.
[13] Soh, S., Byrska, M., Kandere-Grzybowska, K., and Grzybowski, B. A., 2010, "Reaction-Diffusion Systems in Intracellular Molecular Transport and Control," Angew. Chem. Int. Ed. Engl., 49(25), pp. 4170-4198.

[14] Caplan, R. S., and Essig, A., 1999, Bioenergetics and Linear Nonequilibrium Thermodynamics: The Steady State, Harvard University Press, New York.

[15] Demirel, Y., and Sandler, S. I., 2002, "Thermodynamics and Bioenergetics," Biophys. Chem., 97(2-3), pp. 87-111.

[16] Anita, S., and Capasso, V., 2017, "Reaction-Diffusion Systems in Epidemiology," eprint arXiv:1703.02760.

[17] Elias, J., and Clairambault, J., 2014, "Reaction-Diffusion Systems for Spatio-Temporal Intracellular Protein Networks: A Beginners Guide With Two Examples," Comp. Struct. Biotechnol. J., 10(16), pp. 12-22.

[18] Fahmy, E. S., and Abdusalam, H. A., 2009, "Exact Solutions for Some Reaction Diffusion Systems With Nonlinear Reaction Polynomial Terms," Appl. Math. Sci., 3(11), pp. 533-540.

[19] Tuncer, N., Madzvamuse, A and Meir, A. J., 2015, "Projected Finite Elements for Reaction-Diffusion Systems on Stationary Closed Surfaces," Appl. Numer. Math., 96, pp. 45-71.

[20] Stakgold, I., 1979, Green's Functions and Boundary Value Problems, 1st ed., Wiley, New York, Chap. 9.

[21] Taigbenu, A. E., and Onyejekwe, O. O., 1999, "Green's Function-Based Integral Approaches to Nonlinear Transient Boundary-Value Problems (II)," Appl. Math. Model., 23(3), pp. 241-253.

[22] Jones, M. R., and Solovjov, V. P., 2010, "Green's Function Approach to Nonlinear Conduction and Surface Radiation Problems," ASME J. Heat Transfer, 132(2), p. 024502.

[23] Flint, T. F., Francis, J. A., Smith, M. C., and Vasileiou, A. N., 2018, "SemiAnalytical Solutions for the Transient Temperature Fields Induced by a Moving Heat Source in an Orthogonal Domain," Int. J. Therm. Sci., 123, pp. 140-150.

[24] Johansson, B. T., and Lesnic, D., 2008, "A Method of Fundamental Solutions for Transient Heat Conduction," Eng. Anal. Boundary Elem., 32(9), pp. 697-703.

[25] Dong, C. F., 2009, "An Extended Method of Time-Dependent Fundamental Solutions for Inhomogeneous Heat Equation," Eng. Anal. Boundary Elem., 33(5), pp. 717-725.

[26] Yan, L., Yang, F., and Fu, C., 2009, "A Meshless Method for Solving an Inverse Spanwise-Dependent Heat Source Problem,” J. Comput. Phys., 228(1), pp. 123-136.

[27] Axelsson, O., Glushdov, E., and Glushkova, N., 2009, "The Local Greens Function Method in Singularly Perturbed Convection-Diffusion Problems," Math. Comp., 78, pp. 153-170.

[28] Mandaliya, D. D., Moharir, A. S., and Gudi, R. D., 2013, “An Improved Greens Function Method for Isothermal Effectiveness Factor Determination in One- and Two-Dimensional Catalyst Geometries," Chem. Eng. Sci., 91, pp. 197-211.

[29] Lugo-Mendez, H. D., Valdes-Parada, F. J., Porter, M. L., Wood, B. D., and Ochoa-Tapia, J. A., 2015, "Upscaling Diffusion and Nonlinear Reactive Mass Transport in Homogeneous Porous Media," Transp. Porous Med., 107(3), pp. 683-716.

[30] Cole, K. D., Beck, J. V., Woodbury, K. A., and de Monte, F., 2014, “Intrinsic Verification and a Heat Conduction Database," Int. J. Therm. Sci., 78, pp. 36-47.

[31] Demirel, Y., 2006, "Non-Isothermal Reaction-Diffusion System With Thermodynamically Coupled Heat and Mass Transfer," Chem. Eng. Sci., 61(10), pp. 3379-3385.

[32] Cole, K. D., de Monte, F., McMasters, R. L. Woodbury, K. A Haji-Sheikh, A., and Beck, J. V., 2016, "Steady Heat Conduction in Slab Bodies With Generalized Boundary Conditions," International Mechanical Engineering Congress and Exposition, Phoenix, AZ, Nov. 13-16, Paper No. IMECE2016-66605.

[33] Cole, K. D., Beck, J. V., Haji-Shiekh, A., and Litkouhi, B., 2011, Heat Conduction Using Green's Functions, CRC Press, Boca Rotan, FL.

[34] Patankar, S. V., 1980, Numerical Heat and Fluid Flow, McGraw-Hill, New York, Chap. 4.

[35] University of Nebraska-Lincoln, 2017, "EXACT Analytical Conduction Toolbox," University of Nebraska, Lincoln, NE, accessed July 28, 2017, http:// exact.unl.edu

[36] University of Nebraska-Lincoln, 2017, "Green's Function Library," University of Nebraska, Lincoln, NE, accessed July 28, 2017, http://greensfunction.unl.edu

[37] Haji-Sheikh, A., and Beck, J. V., 1990, "Greens Function Partitioning in Galerkin-Based Integral Solution of the Diffusion Equation," ASME J. Heat Transfer, 112(1), pp. 28-34. 